\title{
On the approximation of the boundary layers for the controllability problem of nonlinear singularly perturbed systems
}

\author{
R. Vrabel \\ Institute of Applied Informatics, Automation and Mathematics, Faculty of Materials \\ Science and Technology, Hajdoczyho 1, 91701 Trnava, Slovakia
}

\begin{abstract}
A new systematic approach to the construction of approximate solutions to a class of nonlinear singularly perturbed feedback control systems using the boundary layer functions especially with regard to the possible occurrence of the boundary layers is proposed. For example, problems with feedback control, such as the steady-states of the thermostats, where the controllers add or remove heat, depending upon the temperature registered in another place of the heated bar, can be interpreted with a second-order ordinary differential equation subject to a nonlocal three-point boundary condition. The $O(\epsilon)$ accurate approximation of behavior of these nonlinear systems in terms of the exponentially small boundary layer functions is given. At the end of this paper, we formulate the unsolved controllability problem for nonlinear systems.
\end{abstract}

Keywords: Feedback control, singularly perturbed nonlinear system, boundary layer.

2000 MSC: 93B52, 34B10, 34A34, 34E15, 93C15, 93C70, 34A40

\section{Motivation and introduction}

In various fields of science and engineering, systems with two-time-scale dynamics are often investigated. In state space, such systems are commonly modeled using the mathematical framework of singular perturbations, with a small parameter, say $\epsilon$, determining the degree of separation between the "slow" and "fast" channels of the system. Singularly perturbed systems (SPS) can also occur due to the presence of small "parasitic" parameters, armature inductance in a common model for most DC motors, small time constants, etc.

Singular perturbation problems arise also in heat transfer problem with large Peclet numbers (we often assume $\epsilon$ to be small in order to diminish the effect of diffusion ([23]), Navier-Stokes flows with large Reynolds numbers, chemical

Email address: robert.vrabel@stuba.sk (R. Vrabel) 
reactor theory, aerodynamics, control of reaction-diffusion processes ([8], [20]), quantum mechanics ([1]), optimal control ([24]), for example.

The literature on control of nonlinear SPS is extensive, at least starting with the pioneering work of P. Kokotovic et al. nearly 30 years ago ([18]) and continuing to the present including authors such as Z. Artstein $([2,3]), \mathrm{V}$. Gaitsgory $([4,12,13])$, etc (see, e.g. $[6,7,10,15,22]$ and the references therein).

\section{Problem formulation}

In this paper, we will consider the nonlinear singularly perturbed feedback control system without an outer disturbance of the form

$$
\begin{aligned}
y^{\prime}(t) & =w(t) \\
\epsilon w^{\prime}(t) & =-k y(t)+f(u(t), y(t)) \\
v(t) & =g(y(t))
\end{aligned}
$$

with the required nonlocal boundary conditions

$$
v\left(t_{i}\right)=v\left(t_{m}\right)=v\left(t_{f}\right), \quad t_{i}<t_{m}<t_{f},
$$

where $\epsilon>0$ is a small perturbation parameter, $[y, w]^{T}$ is the state vector, $v(t)$ is the measured output, $u(t)$ is the input control, $k<0$ is a constant and $g$ is a monotone increasing (decreasing) function on $\mathbb{R}$. The state and control variables are not constrained by any boundaries, initial time $t_{i}$ and final time $t_{f}$ are fixed and $y\left(t_{i}\right), y\left(t_{f}\right)$ are free.

Such boundary value problems can arise in the study of the steady-states of a heated bar with the thermostats, where the controllers at $t=t_{i}$ and $t=t_{f}$ maintain a temperature according to the temperature detected by a sensor at $t=t_{m}$. In this case, we consider a uniform bar of length $t_{f}-t_{i}$ with non-uniform temperature lying on the $t$-axis from $t=t_{i}$ to $t=t_{f}$. The parameter $\epsilon$ represents the thermal diffusivity.

Different from [5], in this paper we will not assume that $y\left(t_{i}\right)$ and $y\left(t_{f}\right)$ are fixed and moreover we investigate three-point boundary value problem. There have been some papers considered the multi-point boundary value problems in the literature (see, e.g. [14], [16], [17], [28]) by applying the well known coincidence degree theory and Schauder fixed point theorem or the method of lower and upper solutions. However, there have been fewer papers considered the three-point boundary value problems for SPS without the derivative in the boundary conditions. Recently, in the paper [19], it has been studied the nonlinear system of the form $\epsilon^{2} y^{\prime \prime}=f\left(t, y, y^{\prime}\right), 0<t<1$ subject to the boundary conditions $y(0)=0, y(1)=p y(\tau), 0<\tau<1$ and $p<1$, where the assumption $p<1$ was crucial for proving the main result.

One of the typical behaviors of SPS is the boundary layer phenomenon: the solutions vary rapidly within very thin layer regions near the boundary. The novelty of our approach lies in the introduction of the exponentially small boundary layer functions into the analysis of nonlocal boundary value problems 
and approximation of their solutions. The situation in the case of nonlocal boundary value problem is complicated by the fact that there is an inner point in the boundary conditions, in contrast to the "standard" boundary conditions as the Dirichlet problem, Neumann problem, Robin problem, periodic boundary value problem ([9], [11]), for example. In the problem considered, there does not exist a positive solution $\tilde{\zeta}_{\epsilon}$ of differential equation $\epsilon y^{\prime \prime}-m y=0, m>0$, $\epsilon>0$ (that is, $\tilde{\zeta}_{\epsilon}$ is convex) such that $\tilde{\zeta}_{\epsilon}\left(t_{m}\right)-\tilde{\zeta}_{\epsilon}\left(t_{i}\right)=\eta\left(t_{m}\right)-\eta\left(t_{i}\right)>0$ and $\tilde{\zeta}_{\epsilon}(t) \rightarrow 0^{+}$for $t \in\left(t_{i}, t_{f}\right]$ and $\epsilon \rightarrow 0^{+}$, which could be used to solve this problem by the method of lower and upper solutions and consequently, to approximate the solutions. The application of convex functions is essential for composing the appropriate barrier functions for two-endpoint boundary conditions, see, e.g. [9].

The following assumptions will be made throughout the paper.

A1. For limiting problem (in (2) letting $\left.\epsilon \rightarrow 0^{+}\right) k y=f(u(t), y$ ) there exists $C^{2}$ function $\eta=\eta(t)$ (that is, $\eta$ is continuous up to second derivative) such that $k \eta(t)=f(u(t), \eta(t))$ on $\left[t_{i}, t_{f}\right]$.

Denote $H(\eta)=\left\{(t, y) ; \quad t_{i} \leq t \leq t_{f},|y-\eta(t)|<d(t)\right\}$, where $d(t)$ is the positive continuous function on $\left[t_{i}, t_{f}\right]$ such that

$$
d(t)= \begin{cases}\left|\eta\left(t_{m}\right)-\eta\left(t_{i}\right)\right|+\delta & \text { for } t_{i} \leq t \leq t_{i}+\frac{\delta}{2} \\ \delta & \text { for } t_{i}+\delta \leq t \leq t_{f}-\delta, \\ \left|\eta\left(t_{f}\right)-\eta\left(t_{m}\right)\right|+\delta & \text { for } t_{f}-\frac{\delta}{2} \leq t \leq t_{f}\end{cases}
$$

$\delta$ is a small positive constant.

A2. The function $f \in C^{1}(H(\eta))$ satisfies the condition

$$
\left|\frac{\partial f(u(t), y)}{\partial y}\right| \leq \lambda<-k \quad \text { for every } \quad(t, y) \in H(\eta) .
$$

The assumption (A2) means that the linearization of SPS (1), (2) in a neighbourhood of the set $[\eta(t), 0], t \in\left[t_{i}, t_{f}\right]$, as a set of critical points, has no eigenvalues on the imaginary axis.

In this paper, we characterize the dynamics for slow variable $y$ in a neighborhood of $\eta(t)$ for sufficiently small values of the singular perturbation parameter $\epsilon$ and $t \in\left[t_{i}, t_{f}\right]$. Especially, we focus our attention on the appearance of boundary layers. Moreover, we give the $O(\epsilon)$ accurate approximation of $y$ on $\left[t_{i}, t_{f}\right]$.

Obviously, $y$ is a solution of boundary value problem

$$
\begin{gathered}
\epsilon y^{\prime \prime}(t)+k y(t)=f(u(t), y(t)) \\
y\left(t_{i}\right)=y\left(t_{m}\right)=y\left(t_{f}\right), \quad t_{i}<t_{m}<t_{f} .
\end{gathered}
$$

Recently in [27] we have shown that the solutions of (5), (6), in general, start with fast transient $\left(\left|w_{\epsilon}\left(t_{i}\right)\right| \rightarrow \infty\right)$ of $y_{\epsilon}(t)$ from $y_{\epsilon}\left(t_{i}\right)$ to $\eta(t)$, which is the so-called boundary layer phenomenon, and after decay of this transient they 
remain close to $\eta(t)$ with an arising new fast transient of $y_{\epsilon}(t)$ from $\eta(t)$ to $y_{\epsilon}\left(t_{f}\right)$ $\left(\left|w_{\epsilon}\left(t_{f}\right)\right| \rightarrow \infty\right)$. Boundary layers are formed due to the nonuniform convergence of the exact solution $y_{\epsilon}$ to the degenerate solution $\eta$ in the neighborhood of the ends $t_{i}$ and $t_{f}$ of the considered interval.

\section{Behavior of SPS for $\epsilon \rightarrow 0^{+}$}

Theorem 1 (compare with [27], Theorem 2.1). Under the assumptions (A1) and (A2) there exists $\epsilon_{0}$ such that for every $\epsilon \in\left(0, \epsilon_{0}\right]$ and for every input control $u$ the $S P S(5),(6)$ has in $H(\eta)$ an unique realization, $y_{\epsilon}$, satisfying the inequality

$$
-\zeta_{\epsilon}^{(\operatorname{corr})}(t)-\hat{\zeta}_{\epsilon}(t)-C \epsilon \leq y_{\epsilon}(t)-\left(\eta(t)+\zeta_{\epsilon}(t)\right) \leq \hat{\zeta}_{\epsilon}(t)+C \epsilon
$$

for $\eta\left(t_{m}\right)-\eta\left(t_{i}\right) \geq 0$ and

$$
-\hat{\zeta}_{\epsilon}(t)-C \epsilon \leq y_{\epsilon}(t)-\left(\eta(t)+\zeta_{\epsilon}(t)\right) \leq \zeta_{\epsilon}^{(\operatorname{corr})}(t)+\hat{\zeta}_{\epsilon}(t)+C \epsilon
$$

for $\eta\left(t_{m}\right)-\eta\left(t_{i}\right) \leq 0$ on $\left[t_{i}, t_{f}\right]$ where

$$
\begin{aligned}
\zeta_{\epsilon}(t) & =\frac{\eta\left(t_{m}\right)-\eta\left(t_{i}\right)}{D} \cdot\left(e^{\sqrt{\frac{m}{\epsilon}}\left(t_{f}-t\right)}-e^{\sqrt{\frac{m}{\epsilon}}\left(t-t_{f}\right)}\right. \\
& \left.+e^{\sqrt{\frac{m}{\epsilon}}\left(t-t_{m}\right)}-e^{\sqrt{\frac{m}{\epsilon}}\left(t_{m}-t\right)}\right), \\
\hat{\zeta}_{\epsilon}(t) & =\frac{\left|\eta\left(t_{f}\right)-\eta\left(t_{m}\right)\right|}{D} \cdot\left(e^{\sqrt{\frac{m}{\epsilon}}\left(t-t_{i}\right)}-e^{\sqrt{\frac{m}{\epsilon}}\left(t_{i}-t\right)}\right. \\
& \left.+e^{\sqrt{\frac{m}{\epsilon}}\left(t_{m}-t\right)}-e^{\sqrt{\frac{m}{\epsilon}}\left(t-t_{m}\right)}\right), \\
D & =\left(e^{\sqrt{\frac{m}{\epsilon}}\left(t_{f}-t_{i}\right)}+e^{\sqrt{\frac{m}{\epsilon}}\left(t_{m}-t_{f}\right)}+e^{\sqrt{\frac{m}{\epsilon}}\left(t_{i}-t_{m}\right)}\right) \\
& -\left(e^{\sqrt{\frac{m}{\epsilon}}\left(t_{i}-t_{f}\right)}+e^{\sqrt{\frac{m}{\epsilon}}\left(t_{f}-t_{m}\right)}+e^{\sqrt{\frac{m}{\epsilon}}\left(t_{m}-t_{i}\right)}\right),
\end{aligned}
$$

$m=-k-\lambda, C=\frac{1}{m} \max \left\{\left|\eta^{\prime \prime}(t)\right| ; t \in\left[t_{i}, t_{f}\right]\right\}$ and the positive function

$$
\begin{aligned}
\zeta_{\epsilon}^{(\mathrm{corr})}(t) & =\frac{\lambda\left|\eta\left(t_{m}\right)-\eta\left(t_{i}\right)\right|}{\sqrt{m \epsilon}} \cdot\left[-O(1) \frac{\zeta_{\epsilon}(t)}{\left(\eta\left(t_{m}\right)-\eta\left(t_{i}\right)\right)}\right. \\
& \left.+O\left(e^{\sqrt{\frac{m}{\epsilon}}\left(t_{i}-t_{m}\right)}\right) \frac{\hat{\zeta}_{\epsilon}(t)}{\left|\eta\left(t_{f}\right)-\eta\left(t_{m}\right)\right|}+t O\left(e^{\sqrt{\frac{m}{\epsilon}} \chi(t)}\right)\right],
\end{aligned}
$$

$\chi(t)<0$ for $t \in\left(t_{i}, t_{f}\right]$ and $\zeta_{\epsilon}^{(\text {corr })}\left(t_{i}\right)=\zeta_{\epsilon}^{(\text {corr })}\left(t_{m}\right)$.

We write $s(\epsilon)=O(r(\epsilon))$ when $0<\lim _{\epsilon \rightarrow 0^{+}}\left|\frac{s(\epsilon)}{r(\epsilon)}\right|<\infty$.

The function $\zeta_{\epsilon}(t)$ satisfies

1. $\epsilon \zeta_{\epsilon}^{\prime \prime}-m \zeta_{\epsilon}=0$, 
2. $\zeta_{\epsilon}\left(t_{m}\right)-\zeta_{\epsilon}\left(t_{i}\right)=-\left(\eta\left(t_{m}\right)-\eta\left(t_{i}\right)\right), \zeta_{\epsilon}\left(t_{f}\right)-\zeta_{\epsilon}\left(t_{m}\right)=0$,

3. $\zeta_{\epsilon}(t) \geq 0(\leq 0)$ is decreasing (increasing) for $t_{i} \leq t \leq \frac{t_{f}+t_{m}}{2}$ and increasing (decreasing) for $\frac{t_{f}+t_{m}}{2} \leq t \leq t_{f}$ if $\eta\left(t_{m}\right)-\eta\left(t_{i}\right) \geq 0(\leq 0)$,

4. $\zeta_{\epsilon}(t)$ converges uniformly to 0 for $\epsilon \rightarrow 0^{+}$on every compact subset of $\left(t_{i}, t_{f}\right]$,

5. $\zeta_{\epsilon}(t)=\left(\eta\left(t_{m}\right)-\eta\left(t_{i}\right)\right) O\left(e^{\sqrt{\frac{m}{\epsilon}} \chi(t)}\right)$ where $\chi(t)=t_{i}-t$ for $t_{i} \leq t \leq \frac{t_{f}+t_{m}}{2}$ and $\chi(t)=t-t_{f}+t_{i}-t_{m}$ for $\frac{t_{f}+t_{m}}{2}<t \leq t_{f}$.

The function $\hat{\zeta}_{\epsilon}(t)$ satisfies

1. $\epsilon \hat{\zeta}_{\epsilon}^{\prime \prime}-m \hat{\zeta}_{\epsilon}=0$,

2. $\hat{\zeta}_{\epsilon}\left(t_{m}\right)-\hat{\zeta}_{\epsilon}\left(t_{i}\right)=0, \hat{\zeta}_{\epsilon}\left(t_{f}\right)-\hat{\zeta}_{\epsilon}\left(t_{m}\right)=\left|\eta\left(t_{f}\right)-\eta\left(t_{m}\right)\right|$,

3. $\hat{\zeta}_{\epsilon}(t) \geq 0$ is decreasing for $t_{i} \leq t \leq \frac{t_{i}+t_{m}}{2}$ and increasing for $\frac{t_{i}+t_{m}}{2} \leq t \leq t_{f}$,

4. $\hat{\zeta}_{\epsilon}(t)$ converges uniformly to 0 for $\epsilon \rightarrow 0^{+}$on every compact subset of $\left[t_{i}, t_{f}\right)$,

5. $\hat{\zeta}_{\epsilon}(t)=\left|\eta\left(t_{f}\right)-\eta\left(t_{m}\right)\right| O\left(e^{\sqrt{\frac{m}{\epsilon}} \hat{\chi}(t)}\right)$ where $\hat{\chi}(t)=t-t_{f}$ for $\frac{t_{i}+t_{m}}{2} \leq t \leq t_{f}$ and $\hat{\chi}(t)=t_{m}-t_{f}+t_{i}-t$ for $t_{i} \leq t<\frac{t_{i}+t_{m}}{2}$.

The correction function

$$
\zeta_{\epsilon}^{(\text {corr })}(t)=-\frac{\left(\psi_{\epsilon}\left(t_{i}\right)-\psi_{\epsilon}\left(t_{m}\right)\right)}{\left(\eta\left(t_{m}\right)-\eta\left(t_{i}\right)\right)} \zeta_{\epsilon}(t)+\frac{\left(\psi_{\epsilon}\left(t_{m}\right)-\psi_{\epsilon}\left(t_{f}\right)\right)}{\left|\eta\left(t_{f}\right)-\eta\left(t_{m}\right)\right|} \hat{\zeta}_{\epsilon}(t)+\psi_{\epsilon}(t)
$$

where

$$
\begin{aligned}
\psi_{\epsilon}(t) & =\frac{\lambda\left|\eta\left(t_{m}\right)-\eta\left(t_{i}\right)\right|}{D \sqrt{m \epsilon}} t\left(e^{\sqrt{\frac{m}{\epsilon}}\left(t_{f}-t\right)}+e^{\sqrt{\frac{m}{\epsilon}}\left(t-t_{f}\right)}\right. \\
& \left.-e^{\sqrt{\frac{m}{\epsilon}}\left(t_{m}-t\right)}-e^{\sqrt{\frac{m}{\epsilon}}\left(t-t_{m}\right)}\right)
\end{aligned}
$$

converges uniformly to $0^{+}$on $\left[t_{i}, t_{f}\right]$ for $\epsilon \rightarrow 0^{+}$. and

Theorem 1 implies that $y_{\epsilon}(t)=\eta(t)+O(\epsilon)$ on every compact subset of $\left(t_{i}, t_{f}\right)$

$$
\lim _{\epsilon \rightarrow 0^{+}} y_{\epsilon}\left(t_{i}\right)=\lim _{\epsilon \rightarrow 0^{+}} y_{\epsilon}\left(t_{f}\right)=\lim _{\epsilon \rightarrow 0^{+}} y_{\epsilon}\left(t_{m}\right)=\eta\left(t_{m}\right) .
$$

Consequently,

$$
\lim _{\epsilon \rightarrow 0^{+}} g\left(y_{\epsilon}\left(t_{i}\right)\right)=\lim _{\epsilon \rightarrow 0^{+}} g\left(y_{\epsilon}\left(t_{f}\right)\right)=\lim _{\epsilon \rightarrow 0^{+}} g\left(y_{\epsilon}\left(t_{m}\right)\right)=g\left(\eta\left(t_{m}\right)\right) .
$$

Due to the assumption that $g$ is strictly monotone, the boundary layer effect occurs at the point $t_{i}$ or/and $t_{f}$ in the case when $\eta\left(t_{i}\right) \neq \eta\left(t_{m}\right)$ or/and $\eta\left(t_{f}\right) \neq$ $\eta\left(t_{m}\right)$. 


\section{Approximation of realization of SPS}

The application of numerical methods may give rise to difficulties when the singular perturbation parameter $\epsilon$ tends to zero, especially in the nonlinear case. Then the mesh needs to be refined substantially to grasp the solution within the boundary layers (piecewise uniform mesh of Shishkin-type; see, e.g. [21], [25] and the references therein). The advantage of our approach is that we have to solve only on the parameter $\epsilon$ independent limiting problem $k y=f(u(t), y)$, see the assumption (A1). Then a singular perturbation method is applied to obtain an approximate solution of SPS (5), (6) composed of a solution $\eta$ of reduced problem, small constant and two boundary layer functions to recover the lost nonlocal boundary conditions in the degeneration process.

We use the linear combination of the functions $\eta(t), \zeta_{\epsilon}(t)$ and $\hat{\zeta}_{\epsilon}(t)$ to approximate the exact solution of SPS (5), (6) by the following way. For $\eta\left(t_{f}\right)-\eta\left(t_{m}\right) \leq$ 0 we define the approximate realization $\tilde{y}_{\epsilon}(t)$ of SPS (5), (6) by

$$
\tilde{y}_{\epsilon}(t)=\eta(t)+\zeta_{\epsilon}(t)+\hat{\zeta}_{\epsilon}(t)+C \epsilon
$$

and analogously, for $\eta\left(t_{f}\right)-\eta\left(t_{m}\right) \geq 0$ we define

$$
\tilde{y}_{\epsilon}(t)=\eta(t)+\zeta_{\epsilon}(t)-\hat{\zeta}_{\epsilon}(t)-C \epsilon
$$

where the $\epsilon$-independent constant $C$ is defined in Theorem 1.

It is not difficult to verify that $\tilde{y}_{\epsilon}(t)$ satisfies the boundary conditions (6) and

$$
\lim _{\epsilon \rightarrow 0^{+}} \tilde{y}_{\epsilon}\left(t_{i}\right)=\eta\left(t_{m}\right)=\lim _{\epsilon \rightarrow 0^{+}} \tilde{y}_{\epsilon}\left(t_{f}\right) .
$$

Further,

1. for $\eta\left(t_{f}\right)-\eta\left(t_{m}\right) \leq 0$ and $\eta\left(t_{m}\right)-\eta\left(t_{i}\right) \leq 0$ we obtain the inequality

$$
-\zeta_{\epsilon}^{(\text {corr })}(t) \leq \tilde{y}_{\epsilon}(t)-y_{\epsilon}(t) \leq 2 \hat{\zeta}_{\epsilon}(t)+2 C \epsilon,
$$

2. for $\eta\left(t_{f}\right)-\eta\left(t_{m}\right) \geq 0$ and $\eta\left(t_{m}\right)-\eta\left(t_{i}\right) \geq 0$

$$
-\zeta_{\epsilon}^{(\mathrm{corr})}(t) \leq y_{\epsilon}(t)-\tilde{y}_{\epsilon}(t) \leq 2 \hat{\zeta}_{\epsilon}(t)+2 C \epsilon,
$$

3. for $\eta\left(t_{f}\right)-\eta\left(t_{m}\right) \leq 0$ and $\eta\left(t_{m}\right)-\eta\left(t_{i}\right) \geq 0$

$$
0 \leq \tilde{y}_{\epsilon}(t)-y_{\epsilon}(t) \leq \zeta_{\epsilon}^{(\mathrm{corr})}(t)+2 \hat{\zeta}_{\epsilon}(t)+2 C \epsilon,
$$

4. for $\eta\left(t_{f}\right)-\eta\left(t_{m}\right) \geq 0$ and $\eta\left(t_{m}\right)-\eta\left(t_{i}\right) \leq 0$

$$
0 \leq y_{\epsilon}(t)-\tilde{y}_{\epsilon}(t) \leq \zeta_{\epsilon}^{(\mathrm{corr})}(t)+2 \hat{\zeta}_{\epsilon}(t)+2 C \epsilon .
$$


The right sides of the inequalities (9)-(12) are $O(\epsilon)$ on every compact subset of $\left[t_{i}, t_{f}\right)$. On the other hand, taking into consideration the facts that $\tilde{y}_{\epsilon}\left(t_{i}\right)=$ $\tilde{y}_{\epsilon}\left(t_{f}\right), y_{\epsilon}\left(t_{i}\right)=y_{\epsilon}\left(t_{f}\right)$ and monotonicity of the functions $\zeta_{\epsilon}^{(\text {corr })}(t)+2 \hat{\zeta}_{\epsilon}(t)+2 C \epsilon$ and $2 \hat{\zeta}_{\epsilon}(t)+2 C \epsilon$ with respect to the variable $t$ in a left neighbourhood of $t_{f}$ for small $\epsilon$, we have

$$
\left|y_{\epsilon}(t)-\tilde{y}_{\epsilon}(t)\right| \leq O(\epsilon)
$$

on $\left[t_{i}, t_{f}\right]$, that is, $\tilde{y}_{\epsilon}(t)$ is $O(\epsilon)$ accurate approximation of exact solution $y_{\epsilon}(t)$ of (5), (6) on the whole interval $\left[t_{i}, t_{f}\right]$. We also see that $\left|\tilde{w}_{\epsilon}\left(t_{i}\right)\right| \rightarrow \infty$ and $\left|\tilde{w}_{\epsilon}\left(t_{f}\right)\right| \rightarrow \infty$ for $\epsilon \rightarrow 0^{+}$, where $\tilde{w}_{\epsilon} \equiv \tilde{y}_{\epsilon}^{\prime}$. Thus, $\tilde{y}_{\epsilon}(t)$ is a good approximation of the boundary layers arising in the endpoints of the considered interval $\left[t_{i}, t_{f}\right]$.

We remark that in the special case when $C=0$, that is, if $\eta$ is a first-degree polynomial function or a piecewise linear function (in the second case a small generalization of Theorem 1 is needed) we obtain the exponential convergence rate of $\tilde{y}_{\epsilon}$ to $y_{\epsilon}$ on $\left[t_{i}, t_{f}\right]$ for $\epsilon \rightarrow 0^{+}$.

We remind, that $\tilde{y}_{\epsilon}(t)=\eta(t)$ is not an appropriate approximation of $y_{\epsilon}(t)$ because do not respect the possible appearance of boundary layers.

Consider SPS with quadratic nonlinearity of the form

$$
\epsilon y^{\prime \prime}+k y=y^{2}+u(t), \quad k<0, \quad u \in C^{2}\left(\left[t_{i}, t_{f}\right]\right)
$$

with the boundary conditions (4). The assumptions of Theorem 1 are satisfied if and only if there exists $\lambda>0$ such that

$$
\begin{aligned}
\frac{1}{4}\left(k^{2}-(\lambda-k)^{2}\right) & <u(t)<\frac{1}{4}\left(k^{2}-(\lambda+k)^{2}\right) \quad \text { on } \quad\left[t_{i}, t_{f}\right] \\
\left|u\left(t_{m}\right)-u\left(t_{i}\right)\right| & <\frac{1}{8}\left(\lambda-k-\iota\left(t_{i}\right)\right)\left(\iota\left(t_{i}\right)+\iota\left(t_{m}\right)\right) \\
\left|u\left(t_{f}\right)-u\left(t_{m}\right)\right| & <\frac{1}{8}\left(\lambda-k-\iota\left(t_{f}\right)\right)\left(\iota\left(t_{f}\right)+\iota\left(t_{m}\right)\right) \\
\left|u\left(t_{m}\right)-u\left(t_{i}\right)\right| & <\frac{1}{8}\left(\lambda+k+\iota\left(t_{i}\right)\right)\left(\iota\left(t_{i}\right)+\iota\left(t_{m}\right)\right) \\
\left|u\left(t_{f}\right)-u\left(t_{m}\right)\right| & <\frac{1}{8}\left(\lambda+k+\iota\left(t_{f}\right)\right)\left(\iota\left(t_{f}\right)+\iota\left(t_{m}\right)\right),
\end{aligned}
$$

where $\iota(t)=\sqrt{k^{2}-4 u(t)}$.

For an illustrative example let we consider the problem (13), (4) with $k=-2$, $u(t)=t, t_{i}=0, t_{f}=1 / 2, t_{m}=1 / 4$ and $g=\mathrm{id}$. It is not difficult to verify that the solution $\eta(t)=-1+\sqrt{1-t}$ of reduced problem satisfies the conditions (14)(18) for every $\lambda \in\left(\frac{2}{\sqrt{2}+\sqrt{3}}+2-\sqrt{2}, 2\right)$. Thus, on the basis of Theorem 1 , there exists $\epsilon_{0}=\epsilon_{0}(\lambda)$ such that for every $\epsilon \in\left(0, \epsilon_{0}\right]$ the problem $\epsilon y^{\prime \prime}-2 y=y^{2}+t$, (4) has in $H(\eta)$ the unique solution which is $O(\epsilon)$ close to the approximate solution (7) on $\left[t_{i}, t_{f}\right]$ (Fig. 1), that is, to the function

$$
\tilde{y}_{\epsilon}(t)=-1+\sqrt{1-t}+\zeta_{\epsilon}(t)+\hat{\zeta}_{\epsilon}(t)+\epsilon[(2-\lambda) \sqrt{2}]^{-1} .
$$




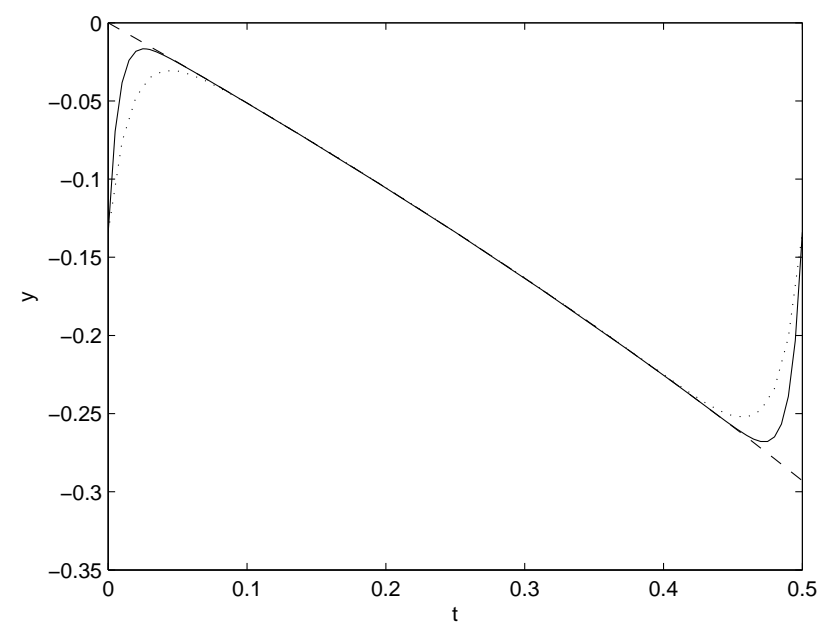

Figure 1: Boundary layer phenomenon for solution of singularly perturbed problem $\epsilon y^{\prime \prime}-2 y=$ $y^{2}+t, y(0)=y(1 / 4)=y(1 / 2)$ (the solid line) with $\epsilon=0.0001$. The dotted and dashed lines represent the approximate solution $\tilde{y}_{\epsilon}(t)$ (with $\lambda=1.6$ ) and solution of reduced problem, the function $\eta(t)=-1+\sqrt{1-t}$, respectively

In the context of previous analysis of the steady-state solutions of 1-D heat transfer equation, it would be interesting to investigate the occurrence of boundary layers for $\epsilon \rightarrow 0^{+}$of perturbed, non-stationary 1-D heat transfer equation, written in the usual form as

$$
\frac{\partial y}{\partial t}=\epsilon \frac{\partial^{2} y}{\partial x^{2}}+k y-f(u(x), y)
$$

subject to the nonlocal boundary conditions

$$
v\left(x_{i}, t\right)=v\left(x_{m}, t\right)=v\left(x_{f}, t\right), \quad x_{i}<x_{m}<x_{f}, \quad t \in[0, \infty),
$$

where $v(x, t)=g(y(x, t))$. The solution $y_{\epsilon}(x, t)$ represents the temperature at point $x$ of the heated bar in the time $t, x \in\left[x_{i}, x_{f}\right], t \in[0, \infty)$. For the initial value problems, the numerical analysis of non-stationary reaction-diffusion systems shows on the presence of boundary layer phenomenon (see, e.g. [26]).

\section{Feedback control of semilinear SPS}

In this section we consider SPS (1), (19), (3) with

$$
\epsilon w^{\prime}(t)=-k y(t)+f(y(t))+u(t)
$$

Let

$$
\left|f^{\prime}(y)\right| \leq \lambda<-k
$$


for $y \in \mathbb{R}$. Moreover, assume that $g \in C^{1}$ and $g_{-1} \in C^{2}$ on $\mathbb{R}$ where $g_{-1}$ denotes an inverse function for $g$.

Now, if $v^{0} \in C^{2}\left(\left[t_{i}, t_{f}\right]\right)$ is desired output of SPS (1), (19), (3) satisfying (4) then it is easy to verify that an adequate feedback control input $u^{0}$ to obtain close $v^{0}$ output is

$$
u^{0}(t)=k g_{-1}\left(v^{0}(t)\right)-f\left(g_{-1}\left(v^{0}(t)\right)\right) .
$$

Hence $\eta^{0}(t)=g_{-1}\left(v^{0}(t)\right)$ and an observable realization $g\left(y_{\epsilon}^{0}\right)$ of system (1), (19), (3) with the boundary condition (4) is $O(\epsilon)$ close to the $g\left(\tilde{y}_{\epsilon}^{0}(t)\right)$.

Indeed, as follows from the Lagrange Theorem and (9)-(12),

$$
\begin{aligned}
\left|g\left(y_{\epsilon}^{0}(t)\right)-g\left(\tilde{y}_{\epsilon}^{0}(t)\right)\right| & \leq \mu\left|y_{\epsilon}^{0}(t)-\eta^{0}(t)\right| \\
& \leq \mu \frac{\epsilon}{m} \max \left\{\left|\eta^{0^{\prime \prime}}(t)\right| ; t \in\left[t_{i}, t_{f}\right]\right\}
\end{aligned}
$$

where $\mu=\max \left\{\left|g^{\prime}(y)\right| ;(t, y) \in H\left(\eta^{0}\right)\right\}$.

\section{Unsolved controllability problem}

Consider the dynamical model described by singularly perturbed differential equation

$$
\epsilon y^{\prime \prime}(t)+\frac{1}{2} \tilde{f}(u(t), y(t))=0,
$$

where $\tilde{f}=2(k y-f) \in C\left(\mathbb{R}^{2}\right)$ (see $\left.(5)\right), u \in C\left(\left[0, t_{f}\right]\right)$ is a continuous control input and $0<\epsilon<<1$ is a singular perturbation parameter. Let $\tilde{f} \neq 0$, and without loss of generality we will assume that $\tilde{f}>0$ and $t_{i}=0$. In this case, the reduced problem $\tilde{f}(u(t), y(t))=0$ does not have a solution $\eta$ (Assumption (A1)), which was the crucial assumption to prove Theorem 1.

Denote by $\left\{t_{i, \epsilon}^{*}\right\}$ the set of turning points in $\left(0, t_{m}\right)$ of exact solutions $y_{\epsilon}$ for problem (20) satisfying $y_{\epsilon}(0)=y_{\epsilon}\left(t_{m}\right)$, that is, $y_{\epsilon}^{\prime}\left(t_{i, \epsilon}^{*}\right)=0$ and $y_{\epsilon}^{\prime \prime}\left(t_{i, \epsilon}^{*}\right) \neq 0$. For the problems considered in the previous sections, the turning points are determined for small $\epsilon$ with sufficient precision by the turning points of the solution $\eta$ of reduced problem. Obviously, for (20) there is only one turning point $t_{\epsilon}^{*}$ of the solution $y_{\epsilon}$ on $\left[0, t_{f}\right]$, and in $t_{\epsilon}^{*}$ acquires its local and global maximum on $\left[0, t_{m}\right]$ and it is possible to steer the control system (20) from the state $y_{\epsilon}(0)$ to the state $y_{\epsilon}\left(t_{m}\right), 0<t_{m}<t_{f}$, satisfying $y_{\epsilon}(0)=y_{\epsilon}\left(t_{m}\right)$ with an arbitrary second boundary condition and for every small $\epsilon$.

Now we will analyze the location of this turning point.

Let consider a special case of $(20)$ when $\tilde{f}(u(t), y(t)) \equiv \tilde{f}\left(u_{0}, y(t)\right)$, that is, the nonlinear mathematical model

$$
\epsilon y^{\prime \prime}(t)+\frac{1}{2} \tilde{f}\left(u_{0}, y(t)\right)=0,
$$

with the initial conditions $y_{\epsilon}(0)=y_{0, \epsilon}, y_{\epsilon}^{\prime}(0)=y_{1, \epsilon}$, where $y_{0, \epsilon}, y_{1, \epsilon}$ are the arbitrary real numbers. Obviously, $y_{1, \epsilon}>0$, because in the case $y_{1, \epsilon} \leq 0$ the 
solution $y_{\epsilon}$ of $(21)$ satisfying $y_{\epsilon}(0)=y_{\epsilon}\left(t_{m}\right)$ has a local minimum at some $t_{0} \in\left(0, t_{m}\right)$ with $y_{\epsilon}^{\prime \prime}\left(t_{0}\right) \geq 0$ which contradicts to the assumption on positivity of the function $\tilde{f}$. Denote by $\tilde{F}_{u_{0}}$ the antiderivative of $\tilde{f}\left(u_{0}, y\right)$, that is, $\tilde{F}_{u_{0}}=$ $\int \tilde{f}\left(u_{0}, y\right) \mathrm{d} y$. The function $\tilde{F}_{u_{0}}$ is strictly increasing and by $\tilde{F}_{u_{0}}^{-1}$ we denote an inverse function to $\tilde{F}_{u_{0}}$. Integrating the differential equation (21) we have

$$
\epsilon\left(y_{\epsilon}^{\prime}(t)\right)^{2}+\tilde{F}_{u_{0}}(y(t))=\epsilon y_{1, \epsilon}^{2}+\tilde{F}_{u_{0}}\left(y_{0, \epsilon}\right) .
$$

Now applying the standard methods we obtain that for every $t \in\left[0, t_{f}\right], y_{\epsilon}(t)$ is an unique root of the equation

$$
\pm 2 \epsilon \int_{\sqrt{\epsilon y_{1, \epsilon}^{2}-\int_{y_{0, \epsilon}}^{y_{\epsilon}(t)} \tilde{f}\left(u_{0}, s\right) \mathrm{d} s}}^{\sqrt{\epsilon y_{1, \epsilon}^{2}}}\left[\tilde{f}\left(\tilde{F}_{u_{0}}^{-1}\left(\tilde{F}_{u_{0}}\left(y_{0, \epsilon}\right)+\epsilon y_{1, \epsilon}^{2}-z^{2}\right)\right)\right]^{-1} \mathrm{~d} z=t
$$

where the sign $+(-)$ on the subintervals of $\left[0, t_{f}\right]$ with $y_{\epsilon}^{\prime} \geq 0\left(y_{\epsilon}^{\prime}<0\right)$, that is, for $t \in\left(0, t_{\epsilon}^{*}\right]\left(t \in\left(t_{\epsilon}^{*}, t_{f}\right]\right)$ is considered, respectively.

Taking into consideration that $y_{\epsilon}^{\prime}\left(t_{\epsilon}^{*}\right)=0$ we have

$$
\tilde{F}_{u_{0}}\left(y\left(t_{\epsilon}^{*}\right)\right)=\epsilon y_{1, \epsilon}^{2}+\tilde{F}_{u_{0}}\left(y_{0, \epsilon}\right) .
$$

Thus for computation of the turning point we obtain from (23) the equation

$$
2 \epsilon \int_{0}^{\sqrt{\epsilon y_{1, \epsilon}^{2}}}\left[\tilde{f}\left(\tilde{F}_{u_{0}}^{-1}\left(\tilde{F}_{u_{0}}\left(y_{0, \epsilon}\right)+\epsilon y_{1, \epsilon}^{2}-z^{2}\right)\right)\right]^{-1} \mathrm{~d} z=t_{\epsilon}^{*} .
$$

To illustrate this theory, let us consider (21) with $\tilde{f}\left(u_{0}, y(t)\right)=e^{y}$. The solution of initial problem is

$$
y_{\epsilon}(t)=\ln \left[c_{1}-c_{1}\left(\frac{e^{\mp \frac{\sqrt{c_{1}}}{\epsilon}\left(t+c_{2}\right)}-1}{e^{\mp \frac{\sqrt{c_{1}}}{\epsilon}}\left(t+c_{2}\right)}+1\right)^{2}\right],
$$

where the sign $-(+)$ on the subintervals of $\left[0, t_{f}\right]$ with $y_{\epsilon}^{\prime} \geq 0\left(y_{\epsilon}^{\prime}<0\right)$ holds, respectively. The constants $c_{1}, c_{2}$ are

$$
c_{1}=\epsilon y_{1, \epsilon}^{2}+\tilde{F}_{u_{0}}\left(y_{0, \epsilon}\right), \quad c_{2}=-\frac{\epsilon}{\sqrt{c_{1}}} \ln \frac{\sqrt{c_{1}}+\sqrt{\epsilon} y_{1, \epsilon}}{\sqrt{c_{1}}-\sqrt{\epsilon} y_{1, \epsilon}}
$$

From (24) we have $y_{\epsilon}\left(t_{\epsilon}^{*}\right)=\ln c_{1}$. Thus, as follows from (25), $t_{\epsilon}^{*}+c_{2}=0$ and we obtain

$$
t_{\epsilon}^{*}=\frac{\epsilon}{\sqrt{c_{1}}} \ln \frac{\sqrt{c_{1}}+\sqrt{\epsilon} y_{1, \epsilon}}{\sqrt{c_{1}}-\sqrt{\epsilon} y_{1, \epsilon}} .
$$


On the other hand, from (25), equating $y_{\epsilon}(0)$ and $y_{\epsilon}\left(t_{m}\right)$ we get $2 c_{2}+t_{m}=0$. Comparing this with (26) we obtain

$$
t_{\epsilon}^{*}=\frac{t_{m}}{2}
$$

The following questions arise in this context:

(i) Where is located the turning point $t_{\epsilon}^{*}$ for nonlinear singularly perturbed system (20) with $\tilde{f}>0$ subject to required boundary condition $y_{\epsilon}(0)=$ $y_{\epsilon}\left(t_{m}\right), 0<t_{m}<t_{f}$ in general? Does have the position independent of singular perturbation parameter $\epsilon$ ?

(ii) Can be controlled a location of turning point by using an appropriate control signal $u$ ?

\section{Acknowledgments}

I would like to express our gratitude to the referees for all the valuable and constructive comments.

\section{References}

[1] J.J. Alvarez, M. Gadella, L.M. Glasser, L.P. Lara, L.M. Nieto: One dimensional systems with singular perturbations, Journal of Physics: Conference Series 284 (2011) 012009. doi:10.1088/1742-6596/284/1/012009

[2] Z. Artstein: Stability in the presence of singular perturbations, Nonlinear Analysis TMA, Vol. 34, No. 6, pp. 817-827, 1998.

[3] Z. Artstein: Singularly perturbed ordinary differential equations with nonautonomous fast dynamics, J. Dynam. Differential Equations, 11, pp. 297318, 1999.

[4] Z. Artstein, V. Gaitsgory: The Value Function of Singularly Perturbed Control System, Applied Mathematics and Optimization, 41, pp. 425-445, 2000 .

[5] I. Boglaev: Robust Monotone Iterates for Nonlinear Singularly Perturbed Boundary Value Problems, Boundary Value Problems Volume 2009, Article ID 320606. doi:10.1155/2009/320606

[6] H. Bouzaouache, N.B. Braiek: On Guaranteed Global Exponential Stability Of Polynomial Singularly Perturbed Control Systems, International Journal of Computers, Communications \& Control, Vol. I, No. 4, pp. 21-34, 2006.

[7] H. Bouzaouache, B.H.B. Ennaceur, M. Benrejeb: Reduced Optimal Control of Nonlinear Singularly Perturbed Systems, Systems Analysis Modelling Simulation, Vol. 43, No. 1, pp. 75-87, 2003. 
[8] C. Castro, E. Zuazua: Unique continuation and control for the heat equation from an oscillating lower dimensional manifold, SIAM Journal on Control and Optimization, Vol. 43, No. 4, pp. 1400-1434, 2004.

[9] K.W. Chang, F.A. Howes: Nonlinear Singular perturbation phenomena: Theory and Applications (Springer-Verlag, New York 1984).

[10] P.D. Christofides, A.R. Teel: Singular Perturbations and Input-to-State Stability, IEEE Trans. Automatic Control, Vol. 41, No. 11, 1996.

[11] C. De Coster, P. Habets: Two-Point Boundary Value Problems: Lower and Upper Solutions, Volume 205 (Mathematics in Science and Engineering, Elsevier Science; 1 edition 2006).

[12] V. Gaitsgory: On a Representation of the Limit Occupational Measures Set of a Control System with Applications to Singularly perturbed Control Systems, SIAM J. on Control and Optimization, Vol. 43, No. 1, pp. 325-340, 2004.

[13] V. Gaitsgory, M.T. Nguyen: Multiscale Singularly Perturbed Control systems: Limit Occupational Measures Sets and Averaging, SIAM Journal on Control and Optimization, Vol. 41, No. 3, pp. 954-974, 2002.

[14] T. Jankowski: Positive solutions of three-point boundary value problems for second order impulsive differential equations with advanced arguments, Applied Mathematics and Computation 197, pp. 179189, 2008.

[15] P. Mei, Ch. Cai, Y. Zou: A Generalized KYP Lemma-Based Approach for $H_{\infty}$ Control of Singularly Perturbed Systems, Circuits, Systems, and Signal Processing, 2009. doi:10.1007/s00034-009-9125-4

[16] R.A. Khan: Positive solutions of four-point singular boundary value problems, Applied Mathematics and Computation, 201, pp. 762-773, 2008.

[17] R.A. Khan, J.R.L. Webb: Existence of at least three solutions of a secondorder three-point boundary value problem, Nonlinear Analysis, 64, pp. 13561366, 2006.

[18] P. Kokotovic, H.K. Khali, J. O'Reilly: Singular Perturbation Methods in Control, Analysis and Design, Academic Press, London, 1986.

[19] X. Lin, W. Liu: Singular perturbation of a second-order three-point boundary value problem for nonlinear systems, J Shanghai Univ (Engl Ed), 13(1), pp. 1619, 2009.

[20] S. Micu, E. Zuazua: Regularity issues for the null-controllability of the linear 1-d heat equation, Systems \& Control Letters, Vol. 60, No. 6, pp. 406-413, 2011. 
[21] J. J. H. Miller, E. ORiordan, G.I. Shishkin: Fitted Numerical Methods for Singular Perturbation Problems: Error Estimates in the Maximum Norm for Linear Problems in One and Two Dimensions, World Scientific, Singapore, 1996.

[22] B. Meng, Y-w. Jing: Robust semiglobally practical stabilization for nonlinear singularly perturbed systems, Nonlinear Analysis 70, pp. 2691-2699, 2009.

[23] A. Munch, E. Zuazua: Numerical approximation of null controls for the heat equation : Ill-posedness and remedies, Inverse Problems, Vol. 26, No. 8, Article Number: 085018, 2010.

[24] T. Nguyen, and Z. Gajic: Finite horizon optimal control of singularly perturbed linear systems: A differential Lyapunov equation approach, IEEE Transactions on Automatic Control, Vol. 55, 21482152, 2010.

[25] E. O' Riordan and J. Quinn: Parameter-uniform numerical methods for some Linear and Nonlinear Singularly Perturbed Convection Diffusion Boundary Turning Point Problems, BIT Numerical Mathematics, 51, pp. 317-337, 2011.

[26] G.I. Shishkin: Grid approximation of singularly perturbed parabolic convection-diffusion equations subject to a piecewise smooth initial condition, Computational Mathematics and Mathematical Physics, Vol. 46, No. 1, 49-72, 2006.

[27] R. Vrabel: Nonlocal Four-Point Boundary Value Problem for the Singularly Perturbed Semilinear Differential Equations, Boundary Value Problems, vol. 2011, Article ID 570493, 9 pages, 2011. doi:10.1155/2011/570493

[28] X. Xu: Positive solutions for singular m-point boundary value problems with positive parameter, J. Math. Anal. Appl., 291, pp. 352-367, 2004. 